Kragujevac Journal of Mathematics

Volume 46(1) (2022), Pages 115-124.

\title{
DIFFERENTIAL SUBORDINATION RESULTS FOR HOLOMORPHIC FUNCTIONS RELATED TO GENERALIZED DIFFERENTIAL OPERATOR
}

\author{
ABBAS KAREEM WANAS ${ }^{1}$
}

\begin{abstract}
In the present investigation, we use the principle of subordination to introduce a new family for holomorphic functions defined by generalized differential operator. Also we establish some interesting geometric properties for functions belonging to this family.
\end{abstract}

\section{Introduction AND PRELIMINARIES}

Let $\mathcal{A}_{m}$ stands for the family of functions $f$ of the form:

$$
f(z)=z+\sum_{n=m+1}^{\infty} a_{n} z^{n} \quad(m \in \mathbb{N}=\{1,2, \ldots\}, z \in U),
$$

which are holomorphic in the open unit disk $U=\{z \in C:|z|<1\}$.

For two functions $f$ and $g$ holomorphic in $U$, we say that the function $f$ is subordinate to $g$, written $f \prec g$ or $f(z) \prec g(z)(z \in U)$, if there exists a Schwarz function $w$ holomorphic in $U$ with $w(0)=0$ and $|w(z)|<1, z \in U$, such that $f(z)=g(w(z))$, $z \in U$. In particular, if the function $g$ is univalent in $U$, then $f \prec g$ if and only if $f(0)=g(0)$ and $f(U) \subset g(U)$ (see [6]).

If $f \in \mathcal{A}_{m}$ is given by (1.1) and $g \in \mathcal{A}_{m}$ given by

$$
g(z)=z+\sum_{n=m+1}^{\infty} b_{n} z^{n} \quad(m \in \mathbb{N}=\{1,2, \ldots\}, z \in U)
$$

Key words and phrases. Holomorphic functions, Differential subordination, Convex univalent, Hadamard product, Generalized differential operator.

2010 Mathematics Subject Classification. Primary: 30C45. Secondary: 30A20.

DOI 10.46793/KgJMat2201.115W

Received: March 29, 2019.

Accepted: September 06, 2019. 
then the Hadamard product (or convolution) $f * g$ of $f$ and $g$ is defined by

$$
(f * g)(z)=z+\sum_{n=m+1}^{\infty} a_{n} b_{n} z^{n}=(g * f)(z) .
$$

A function $f \in \mathcal{A}_{m}$ is said to be starlike of order $\rho$ in $U$ if

$$
\operatorname{Re}\left\{\frac{z f^{\prime}(z)}{f(z)}\right\}>\rho \quad(0 \leq \rho<1, z \in U) .
$$

Indicate the class of all starlike functions of order $\rho$ in $U$ by $S^{*}(\rho)$.

A function $f \in \mathcal{A}_{m}$ is said to be prestarlike of order $\rho$ in $U$ if

$$
\frac{z}{(1-z)^{2(1-\rho)}} * f(z) \in S^{*}(\rho) \quad(\rho<1)
$$

Indicate the class of all prestarlike functions of order $\rho$ in $U$ by $\operatorname{Re}(\rho)$.

Clearly a function $f \in \mathcal{A}_{m}$ is in the class $\operatorname{Re}(0)$ if and only if $f$ is convex univalent in $U$ and $\operatorname{Re}\left(\frac{1}{2}\right)=S^{*}\left(\frac{1}{2}\right)$.

For $\sigma \in N_{0}=N \cup\{0\}, \alpha, \delta \geq 0, \tau, \lambda, \beta>0$ and $\alpha \neq \lambda$, we consider the generalized differential operator $A_{\tau, \lambda, \delta}^{\sigma}(\alpha, \beta): \mathcal{A}_{m} \rightarrow \mathcal{A}_{m}$, introduced by Amourah and Darus [2], where

$$
A_{\tau, \lambda, \delta}^{\sigma}(\alpha, \beta) f(z)=z+\sum_{n=m+1}^{\infty}\left[1+\frac{(n-1)((\lambda-\alpha) \beta+n \delta)}{\tau+\lambda}\right]^{\sigma} a_{n} z^{n} .
$$

It is readily verified from (1.2) that

$$
\begin{aligned}
z\left(A_{\tau, \lambda, \delta}^{\sigma}(\alpha, \beta) f(z)\right)^{\prime}= & \frac{\tau+\lambda}{(\lambda-\alpha) \beta+n \delta} A_{\tau, \lambda, \delta}^{\sigma+1}(\alpha, \beta) f(z) \\
& +\left(1-\frac{\tau+\lambda}{(\lambda-\alpha) \beta+n \delta}\right) A_{\tau, \lambda, \delta}^{\sigma}(\alpha, \beta) f(z) .
\end{aligned}
$$

Here, we would point out some of the special cases of the operator defined by (1.2) can be found in $[1,4,5,11]$.

Let $H$ be the class of functions $h$ with $h(0)=1$, which are holomorphic and convex univalent in $U$.

Definition 1.1. A function $f \in \mathcal{A}_{m}$ is said to be in the class $\mathcal{M}(\eta, \sigma, \tau, \lambda, \delta, \alpha, \beta, m ; h)$ if it satisfies the subordination condition:

$$
\frac{1}{z}\left[\left(1-\frac{\eta(\tau+\lambda)}{(\lambda-\alpha) \beta+n \delta}\right) A_{\tau, \lambda, \delta}^{\sigma}(\alpha, \beta) f(z)+\frac{\eta(\tau+\lambda)}{(\lambda-\alpha) \beta+n \delta} A_{\tau, \lambda, \delta}^{\sigma+1}(\alpha, \beta) f(z)\right] \prec h(z),
$$

where $\eta \in C, \sigma \in N_{0}=N \cup\{0\}, \alpha, \delta \geq 0, \tau, \lambda, \beta>0, \alpha \neq \lambda$ and $h \in H$.

Now, we need the following lemmas that will be used to prove our main results. 
Lemma $1.1([8])$. Let $g$ be holomorphic in $U$ and let $h$ be holomorphic and convex univalent in $U$ with $h(0)=g(0)$. If

$$
g(z)+\frac{1}{\mu} z g^{\prime}(z) \prec h(z),
$$

where $\operatorname{Re}(\mu) \geq 0$ and $\mu \neq 0$, then

$$
g(z) \prec \breve{h}(z)=\mu z^{-\mu} \int_{0}^{z} t^{\mu-1} h(t) d t \prec h(z)
$$

and $\breve{h}$ is the best dominant of (1.5).

Lemma $1.2([10])$. Let $\rho<1, f \in S^{*}(\rho)$ and $g \in \operatorname{Re}(\rho)$. Then, for any holomorphic function $F$ in $U$

$$
\frac{g *(f F)}{g * f}(U) \subset \overline{c o}(F(U)),
$$

where $\overline{c o}(F(U))$ denotes the closed convex hull of $F(U)$.

Such type of study was carried out by various authors for another classes, like, Liu [7], Prajapat and Raina [9], Atshan and Wanas [3], Wanas [12] and Wanas and Majeed [13].

\section{Main Results}

Theorem 2.1. Let $0 \leq \eta<\varepsilon$. Then

$$
\mathcal{M}(\varepsilon, \sigma, \tau, \lambda, \delta, \alpha, \beta, m ; h) \subset \mathcal{M}(\eta, \sigma, \tau, \lambda, \delta, \alpha, \beta, m ; h) .
$$

Proof. Let $0 \leq \eta<\varepsilon$ and $f \in \mathcal{M}(\varepsilon, \sigma, \tau, \lambda, \delta, \alpha, \beta, m ; h)$. Assume that

$$
g(z)=\frac{A_{\tau, \lambda, \delta}^{\sigma}(\alpha, \beta) f(z)}{z}=1+\sum_{n=m+1}^{\infty}\left[1+\frac{(n-1)((\lambda-\alpha) \beta+n \delta)}{\tau+\lambda}\right]^{\sigma} a_{n} z^{n-1} .
$$

It is obvious that the function $g$ is holomorphic in $U$ and $g(0)=1$. Since $f \in$ $\mathcal{M}(\varepsilon, \sigma, \tau, \lambda, \delta, \alpha, \beta, m ; h)$, then we deduce that

$$
\frac{1}{z}\left[\left(1-\frac{\varepsilon(\tau+\lambda)}{(\lambda-\alpha) \beta+n \delta}\right) A_{\tau, \lambda, \delta}^{\sigma}(\alpha, \beta) f(z)+\frac{\varepsilon(\tau+\lambda)}{(\lambda-\alpha) \beta+n \delta} A_{\tau, \lambda, \delta}^{\sigma+1}(\alpha, \beta) f(z)\right] \prec h(z) .
$$

Differentiating both sides of (2.1) with respect to $z$ and using (1.3) and (2.2), we find that

$$
\begin{aligned}
& \frac{1}{z}\left[\left(1-\frac{\varepsilon(\tau+\lambda)}{(\lambda-\alpha) \beta+n \delta}\right) A_{\tau, \lambda, \delta}^{\sigma}(\alpha, \beta) f(z)+\frac{\varepsilon(\tau+\lambda)}{(\lambda-\alpha) \beta+n \delta} A_{\tau, \lambda, \delta}^{\sigma+1}(\alpha, \beta) f(z)\right] \\
= & \frac{1}{z}\left[(1-\varepsilon) A_{\tau, \lambda, \delta}^{\sigma}(\alpha, \beta) f(z)+\varepsilon\left(1-\frac{\tau+\lambda}{(\lambda-\alpha) \beta+n \delta}\right) A_{\tau, \lambda, \delta}^{\sigma}(\alpha, \beta) f(z)\right. \\
& \left.+\frac{\varepsilon(\tau+\lambda)}{(\lambda-\alpha) \beta+n \delta} A_{\tau, \lambda, \delta}^{\sigma+1}(\alpha, \beta) f(z)\right]
\end{aligned}
$$




$$
\begin{aligned}
& =(1-\varepsilon) \frac{A_{\tau, \lambda, \delta}^{\sigma}(\alpha, \beta) f(z)}{z}+\varepsilon\left(A_{\tau, \lambda, \delta}^{\sigma}(\alpha, \beta) f(z)\right)^{\prime} \\
& =\frac{A_{\tau, \lambda, \delta}^{\sigma}(\alpha, \beta) f(z)}{z}+\varepsilon z\left(\frac{A_{\tau, \lambda, \delta}^{\sigma}(\alpha, \beta) f(z)}{z}\right)^{\prime} \\
& =g(z)+\varepsilon z g^{\prime}(z) \prec h(z) .
\end{aligned}
$$

An application of Lemma 1.1 with $\mu=\frac{1}{\varepsilon}$, yields

$$
g(z) \prec h(z) .
$$

Evidently, $0 \leq \frac{\eta}{\varepsilon}<1$ and that $h$ is convex univalent in $U$, it follows from (2.1), (2.2) and (2.3) that

$$
\begin{aligned}
& \frac{1}{z}\left[\left(1-\frac{\eta(\tau+\lambda)}{(\lambda-\alpha) \beta+n \delta}\right) A_{\tau, \lambda, \delta}^{\sigma}(\alpha, \beta) f(z)+\frac{\eta(\tau+\lambda)}{(\lambda-\alpha) \beta+n \delta} A_{\tau, \lambda, \delta}^{\sigma+1}(\alpha, \beta) f(z)\right] \\
= & \frac{\eta}{\varepsilon z}\left[\left(1-\frac{\varepsilon(\tau+\lambda)}{(\lambda-\alpha) \beta+n \delta}\right) A_{\tau, \lambda, \delta}^{\sigma}(\alpha, \beta) f(z)+\frac{\varepsilon(\tau+\lambda)}{(\lambda-\alpha) \beta+n \delta} A_{\tau, \lambda, \delta}^{\sigma+1}(\alpha, \beta) f(z)\right] \\
& +\left(1-\frac{\eta}{\varepsilon}\right) g(z) \prec h(z) .
\end{aligned}
$$

Hence, $f \in \mathcal{M}(\eta, \sigma, \tau, \lambda, \delta, \alpha, \beta, m ; h)$ and the proof of Theorem 2.1 is completed.

Theorem 2.2. Let $\operatorname{Re}\left\{\frac{\tau+\lambda}{(\lambda-\alpha) \beta+n \delta}\right\} \geq 0$ and $\frac{\tau+\lambda}{(\lambda-\alpha) \beta+n \delta} \neq 0$. Then

$$
\mathcal{M}(\eta, \sigma+1, \tau, \lambda, \delta, \alpha, \beta, m ; h) \subset \mathcal{M}(\eta, \sigma, \tau, \lambda, \delta, \alpha, \beta, m ; h) .
$$

Proof. Let $f \in \mathcal{M}(\eta, \sigma+1, \tau, \lambda, \delta, \alpha, \beta, m ; h)$ and suppose that

$$
g(z)=\frac{1}{z}\left[\left(1-\frac{\eta(\tau+\lambda)}{(\lambda-\alpha) \beta+n \delta}\right) A_{\tau, \lambda, \delta}^{\sigma}(\alpha, \beta) f(z)+\frac{\eta(\tau+\lambda)}{(\lambda-\alpha) \beta+n \delta} A_{\tau, \lambda, \delta}^{\sigma+1}(\alpha, \beta) f(z)\right] .
$$

By taking the derivatives in the both sides of (2.4) with respect to $z$ and using (1.3), we conclude that

$$
\begin{aligned}
& g(z)+z g^{\prime}(z) \\
= & \frac{1}{z}\left[\left(1-\frac{\eta(\tau+\lambda)}{(\lambda-\alpha) \beta+n \delta}\right)\left(1-\frac{\tau+\lambda}{(\lambda-\alpha) \beta+n \delta}\right) A_{\tau, \lambda, \delta}^{\sigma}(\alpha, \beta) f(z)\right. \\
& +\left(1+\eta\left(1-\frac{2(\tau+\lambda)}{(\lambda-\alpha) \beta+n \delta}\right)\right) \frac{\tau+\lambda}{(\lambda-\alpha) \beta+n \delta} A_{\tau, \lambda, \delta}^{\sigma+1}(\alpha, \beta) f(z) \\
& \left.+\eta\left(\frac{\tau+\lambda}{(\lambda-\alpha) \beta+n \delta}\right)^{2} A_{\tau, \lambda, \delta}^{\sigma+2}(\alpha, \beta) f(z)\right] .
\end{aligned}
$$

In the light of (2.4) and (2.5), we deduce that

$$
\frac{\tau+\lambda}{(\lambda-\alpha) \beta+n \delta} g(z)+z g^{\prime}(z)
$$




$$
\begin{aligned}
= & \frac{1}{z}\left[\left(1-\frac{\eta(\tau+\lambda)}{(\lambda-\alpha) \beta+n \delta}\right)\left(\frac{\tau+\lambda}{(\lambda-\alpha) \beta+n \delta}\right) A_{\tau, \lambda, \delta}^{\sigma+1}(\alpha, \beta) f(z)\right. \\
& \left.+\eta\left(\frac{\tau+\lambda}{(\lambda-\alpha) \beta+n \delta}\right)^{2} A_{\tau, \lambda, \delta}^{\sigma+2}(\alpha, \beta) f(z)\right],
\end{aligned}
$$

that is

$$
\begin{aligned}
g(z)+\frac{(\lambda-\alpha) \beta+n \delta}{\tau+\lambda} z g^{\prime}(z)= & \frac{1}{z}\left[\left(1-\frac{\eta(\tau+\lambda)}{(\lambda-\alpha) \beta+n \delta}\right) A_{\tau, \lambda, \delta}^{\sigma+1}(\alpha, \beta) f(z)\right. \\
& \left.+\frac{\eta(\tau+\lambda)}{(\lambda-\alpha) \beta+n \delta} A_{\tau, \lambda, \delta}^{\sigma+2}(\alpha, \beta) f(z)\right]
\end{aligned}
$$

Since $f \in \mathcal{M}(\eta, \sigma+1, \tau, \lambda, \delta, \alpha, \beta, m ; h)$, then it follows from (2.6) that

$$
g(z)+\frac{(\lambda-\alpha) \beta+n \delta}{\tau+\lambda} z g^{\prime}(z) \prec h(z),
$$

where

$$
\operatorname{Re}\left\{\frac{\tau+\lambda}{(\lambda-\alpha) \beta+n \delta}\right\} \geq 0, \quad \frac{\tau+\lambda}{(\lambda-\alpha) \beta+n \delta} \neq 0 .
$$

An application of Lemma 1.1, with $\mu=\frac{\tau+\lambda}{(\lambda-\alpha) \beta+n \delta}$, yields $g(z) \prec h(z)$. In view of (2.4), we have

$$
\frac{1}{z}\left[\left(1-\frac{\eta(\tau+\lambda)}{(\lambda-\alpha) \beta+n \delta}\right) A_{\tau, \lambda, \delta}^{\sigma}(\alpha, \beta) f(z)+\frac{\eta(\tau+\lambda)}{(\lambda-\alpha) \beta+n \delta} A_{\tau, \lambda, \delta}^{\sigma+1}(\alpha, \beta) f(z)\right] \prec h(z) .
$$

This shows that $f \in \mathcal{M}(\eta, \sigma, \tau, \lambda, \delta, \alpha, \beta, m ; h)$ and the proof of Theorem 2.2 is completed.

Theorem 2.3. Let $\eta>0, \gamma>0$ and $f \in \mathcal{M}(\eta, \sigma, \tau, \lambda, \delta, \alpha, \beta, m ; \gamma h+1-\gamma)$. If $\gamma \leq \gamma_{0}$, where

$$
\gamma_{0}=\frac{1}{2}\left(1-\frac{1}{\eta} \int_{0}^{1} \frac{u^{\frac{1}{\eta}-1}}{1+u} d u\right)^{-1}
$$

then $f \in \mathcal{M}(0, \sigma, \tau, \lambda, \delta, \alpha, \beta, m ; h)$. The bound $\gamma_{0}$ is the sharp when $h(z)=\frac{1}{1-z}$.

Proof. Assume that

$$
g(z)=\frac{A_{\tau, \lambda, \delta}^{\sigma}(\alpha, \beta) f(z)}{z}
$$

Let $f \in \mathcal{M}(\eta, \sigma, \tau, \lambda, \delta, \alpha, \beta, m ; \gamma h+1-\gamma)$ with $\eta>0$ and $\gamma>0$. Then we obtain

$$
\begin{aligned}
& g(z)+\eta z g^{\prime}(z) \\
= & \frac{1}{z}\left[\left(1-\frac{\eta(\tau+\lambda)}{(\lambda-\alpha) \beta+n \delta}\right) A_{\tau, \lambda, \delta}^{\sigma}(\alpha, \beta) f(z)+\frac{\eta(\tau+\lambda)}{(\lambda-\alpha) \beta+n \delta} A_{\tau, \lambda, \delta}^{\sigma+1}(\alpha, \beta) f(z)\right] \\
\prec & \gamma h(z)+1-\gamma .
\end{aligned}
$$


Making use of Lemma 1.1, we observe that

$$
g(z) \prec \frac{\gamma}{\eta} z^{-\frac{1}{\eta}} \int_{0}^{z} t^{\frac{1}{\eta}-1} h(t) d t+1-\gamma=(h * \phi)(z),
$$

where

$$
\phi(z)=\frac{\gamma}{\eta} z^{-\frac{1}{\eta}} \int_{0}^{z} \frac{t^{\frac{1}{\eta}-1}}{1-t} d t+1-\gamma .
$$

If $0<\gamma \leq \gamma_{0}$, where $\gamma_{0}>1$ is given by (2.7), then we find from (2.10) that

(2.11) $\operatorname{Re}(\phi(z))=\frac{\gamma}{\eta} \int_{0}^{1} u^{\frac{1}{\eta}-1} \operatorname{Re}\left(\frac{1}{1-u z}\right) d u+1-\gamma>\frac{\gamma}{\eta} \int_{0}^{1} \frac{u^{\frac{1}{\eta}-1}}{1+u} d u+1-\gamma \geq \frac{1}{2}$.

By using (2.8) and (2.9), we have

$$
\frac{A_{\tau, \lambda, \delta}^{\sigma}(\alpha, \beta) f(z)}{z} \prec(h * \phi)(z) .
$$

In the light of (2.11), we note that the function $\phi(z)$ has the Herglotz representation

$$
\phi(z)=\int_{|x|=1} \frac{d \mu(x)}{1-x z} \quad(z \in U)
$$

where $\mu(x)$ is a probability measure defined on the unit circle $|x|=1$ and

$$
\int_{|x|=1} d \mu(x)=1
$$

Since $h$ is convex univalent in $U$, then we deduce from (2.12) and (2.13) that

$$
\frac{A_{\tau, \lambda, \delta}^{\sigma}(\alpha, \beta) f(z)}{z} \prec(h * \phi)(z)=\int_{|x|=1} \phi(x z) d \mu(x) \prec h(z) .
$$

This shows that $f \in \mathcal{M}(0, \sigma, \tau, \lambda, \delta, \alpha, \beta, m ; h)$. For $h(z)=\frac{1}{1-z}$ and $f \in \mathcal{A}_{m}$ defined by

$$
\frac{A_{\tau, \lambda, \delta}^{\sigma}(\alpha, \beta) f(z)}{z}=\frac{\gamma}{\eta} z^{-\frac{1}{\eta}} \int_{0}^{z} \frac{t^{\frac{1}{\eta}-1}}{1-t} d t+1-\gamma
$$

we obtain

$$
\begin{aligned}
& \frac{1}{z}\left[\left(1-\frac{\eta(\tau+\lambda)}{(\lambda-\alpha) \beta+n \delta}\right) A_{\tau, \lambda, \delta}^{\sigma}(\alpha, \beta) f(z)+\frac{\eta(\tau+\lambda)}{(\lambda-\alpha) \beta+n \delta} A_{\tau, \lambda, \delta}^{\sigma+1}(\alpha, \beta) f(z)\right] \\
= & \gamma h(z)+1-\gamma .
\end{aligned}
$$

Thus, $f \in \mathcal{M}(\eta, \sigma, \tau, \lambda, \delta, \alpha, \beta, m ; \gamma h+1-\gamma)$. Also, for $\gamma>\gamma_{0}$, we have

$$
\operatorname{Re}\left\{\frac{A_{\tau, \lambda, \delta}^{\sigma}(\alpha, \beta) f(z)}{z}\right\} \rightarrow \frac{\gamma}{\eta} \int_{0}^{1} \frac{u^{\frac{1}{\eta}-1}}{1+u} d u+1-\gamma<\frac{1}{2} \quad(z \rightarrow-1),
$$

which implies that $f \notin \mathcal{M}(0, \sigma, \tau, \lambda, \delta, \alpha, \beta, m ; h)$. Thus, the bound $\gamma_{0}$ cannot be increased when $h(z)=\frac{1}{1-z}$. This completes the proof of the theorem. 
Theorem 2.4. Let $f \in \mathcal{M}(\eta, \sigma, \tau, \lambda, \delta, \alpha, \beta, m ; h)$ be defined as in (1.1). Then the function I defined by

$$
I(z)=\frac{c+1}{z^{c}} \int_{0}^{z} t^{c-1} f(t) d t \quad(\operatorname{Re}(c)>-1)
$$

is also in the class $\mathcal{M}(\eta, \sigma, \tau, \lambda, \delta, \alpha, \beta, m ; h)$.

Proof. Let $f \in \mathcal{M}(\eta, \sigma, \tau, \lambda, \delta, \alpha, \beta, m ; h)$ be defined as in (1.1). Then, we find that (2.14)

$\frac{1}{z}\left[\left(1-\frac{\eta(\tau+\lambda)}{(\lambda-\alpha) \beta+n \delta}\right) A_{\tau, \lambda, \delta}^{\sigma}(\alpha, \beta) f(z)+\frac{\eta(\tau+\lambda)}{(\lambda-\alpha) \beta+n \delta} A_{\tau, \lambda, \delta}^{\sigma+1}(\alpha, \beta) f(z)\right] \prec h(z)$.

We can easily see that

$$
I(z)=\frac{c+1}{z^{c}} \int_{0}^{z} t^{c-1} f(t) d t=z+\sum_{n=m+1}^{\infty} \frac{c+1}{c+n} a_{n} z^{n} .
$$

We have from (2.15) that $I \in \mathcal{A}_{m}$ and

$$
f(z)=\frac{c I(z)+z I^{\prime}(z)}{c+1} .
$$

Define the function $J$ by

$$
J(z)=\frac{1}{z}\left[\left(1-\frac{\eta(\tau+\lambda)}{(\lambda-\alpha) \beta+n \delta}\right) A_{\tau, \lambda, \delta}^{\sigma}(\alpha, \beta) I(z)+\frac{\eta(\tau+\lambda)}{(\lambda-\alpha) \beta+n \delta} A_{\tau, \lambda, \delta}^{\sigma+1}(\alpha, \beta) I(z)\right] .
$$

Differentiating both sides of (2.17) with respect to $z$ and using (2.14) and (2.16), we obtain

$$
\begin{aligned}
& \frac{1}{z}\left[\left(1-\frac{\eta(\tau+\lambda)}{(\lambda-\alpha) \beta+n \delta}\right) A_{\tau, \lambda, \delta}^{\sigma}(\alpha, \beta) f(z)+\frac{\eta(\tau+\lambda)}{(\lambda-\alpha) \beta+n \delta} A_{\tau, \lambda, \delta}^{\sigma+1}(\alpha, \beta) f(z)\right] \\
= & \frac{1}{z}\left[\left(1-\frac{\eta(\tau+\lambda)}{(\lambda-\alpha) \beta+n \delta}\right) A_{\tau, \lambda, \delta}^{\sigma}(\alpha, \beta)\left(\frac{c I(z)+z I^{\prime}(z)}{c+1}\right)\right. \\
& \left.+\frac{\eta(\tau+\lambda)}{(\lambda-\alpha) \beta+n \delta} A_{\tau, \lambda, \delta}^{\sigma+1}(\alpha, \beta)\left(\frac{c I(z)+z I^{\prime}(z)}{c+1}\right)\right] \\
= & \frac{c}{z(c+1)}\left[\left(1-\frac{\eta(\tau+\lambda)}{(\lambda-\alpha) \beta+n \delta}\right) A_{\tau, \lambda, \delta}^{\sigma}(\alpha, \beta) I(z)\right. \\
& \left.+\frac{\eta(\tau+\lambda)}{(\lambda-\alpha) \beta+n \delta} A_{\tau, \lambda, \delta}^{\sigma+1}(\alpha, \beta) I(z)\right] \\
& +\frac{1}{z(c+1)}\left[\left(1-\frac{\eta(\tau+\lambda)}{(\lambda-\alpha) \beta+n \delta}\right) A_{\tau, \lambda, \delta}^{\sigma}(\alpha, \beta)\left(z I^{\prime}(z)\right)\right. \\
& \left.+\frac{\eta(\tau+\lambda)}{(\lambda-\alpha) \beta+n \delta} A_{\tau, \lambda, \delta}^{\sigma+1}(\alpha, \beta)\left(z I^{\prime}(z)\right)\right] \\
= & \frac{c}{c+1} J(z)+\frac{1}{c+1}\left(z J^{\prime}(z)+J(z)\right)=J(z)+\frac{1}{c+1} z J^{\prime}(z) \prec h(z) .
\end{aligned}
$$


An application of Lemma 1.1 with $\mu=c+1$, yields $J(z) \prec h(z)$. By using (2.17), we get

$$
\frac{1}{z}\left[\left(1-\frac{\eta(\tau+\lambda)}{(\lambda-\alpha) \beta+n \delta}\right) A_{\tau, \lambda, \delta}^{\sigma}(\alpha, \beta) I(z)+\frac{\eta(\tau+\lambda)}{(\lambda-\alpha) \beta+n \delta} A_{\tau, \lambda, \delta}^{\sigma+1}(\alpha, \beta) I(z)\right] \prec h(z),
$$

which implies that $I \in \mathcal{M}(\eta, \sigma, \tau, \lambda, \delta, \alpha, \beta, m ; h)$.

Theorem 2.5. Let $f \in \mathcal{M}(\eta, \sigma, \tau, \lambda, \delta, \alpha, \beta, m ; h), g \in \mathcal{A}_{m}$ and

$$
\operatorname{Re}\left\{\frac{g(z)}{z}\right\}>\frac{1}{2}
$$

Then $f * g \in \mathcal{M}(\eta, \sigma, \tau, \lambda, \delta, \alpha, \beta, m ; h)$.

Proof. Let $f \in \mathcal{M}(\eta, \sigma, \tau, \lambda, \delta, \alpha, \beta, m ; h)$ and $g \in \mathcal{A}_{m}$. Then, we have

$$
\begin{aligned}
& \frac{1}{z}\left[\left(1-\frac{\eta(\tau+\lambda)}{(\lambda-\alpha) \beta+n \delta}\right) A_{\tau, \lambda, \delta}^{\sigma}(\alpha, \beta)(f * g)(z)\right. \\
& \left.+\frac{\eta(\tau+\lambda)}{(\lambda-\alpha) \beta+n \delta} A_{\tau, \lambda, \delta}^{\sigma+1}(\alpha, \beta)(f * g)(z)\right] \\
& =\left(1-\frac{\eta(\tau+\lambda)}{(\lambda-\alpha) \beta+n \delta}\right)\left(\frac{g(z)}{z}\right) *\left(\frac{A_{\tau, \lambda, \delta}^{\sigma}(\alpha, \beta) f(z)}{z}\right) \\
& +\frac{\eta(\tau+\lambda)}{(\lambda-\alpha) \beta+n \delta}\left(\frac{g(z)}{z}\right) *\left(\frac{A_{\tau, \lambda, \delta}^{\sigma+1}(\alpha, \beta) f(z)}{z}\right)=\left(\frac{g(z)}{z}\right) * \varphi(z),
\end{aligned}
$$

where

$$
\begin{aligned}
\varphi(z) & =\frac{1}{z}\left[\left(1-\frac{\eta(\tau+\lambda)}{(\lambda-\alpha) \beta+n \delta}\right) A_{\tau, \lambda, \delta}^{\sigma}(\alpha, \beta) f(z)+\frac{\eta(\tau+\lambda)}{(\lambda-\alpha) \beta+n \delta} A_{\tau, \lambda, \delta}^{\sigma+1}(\alpha, \beta) f(z)\right] \\
& \prec h(z) .
\end{aligned}
$$

In view of (2.18), we note that the function $\frac{g(z)}{z}$ has the Herglotz representation

$$
\frac{g(z)}{z}=\int_{|x|=1} \frac{d \mu(x)}{1-x z} \quad(z \in U)
$$

where $\mu(x)$ is a probability measure defined on the unit circle $|x|=1$ and

$$
\int_{|x|=1} d \mu(x)=1
$$

Since $h$ is convex univalent in $U$, then we find from (2.19), (2.20) and (2.21) that

$$
\begin{aligned}
& \frac{1}{z}\left[\left(1-\frac{\eta(\tau+\lambda)}{(\lambda-\alpha) \beta+n \delta}\right) A_{\tau, \lambda, \delta}^{\sigma}(\alpha, \beta)(f * g)(z)+\frac{\eta(\tau+\lambda)}{(\lambda-\alpha) \beta+n \delta}\right. \\
& \left.\quad \times A_{\tau, \lambda, \delta}^{\sigma+1}(\alpha, \beta)(f * g)(z)\right]
\end{aligned}
$$




$$
=\int_{|x|=1} \varphi(x z) d \mu(x) \prec h(z) .
$$

This shows that $f * g \in \mathcal{M}(\eta, \sigma, \tau, \lambda, \delta, \alpha, \beta, m ; h)$.

Theorem 2.6. Let $f \in \mathcal{M}(\eta, \sigma, \tau, \lambda, \delta, \alpha, \beta, m ; h)$ and $g \in \mathcal{A}_{m}$ be prestarlike of order $\alpha,(\alpha<1)$. Then $f * g \in \mathcal{M}(\eta, \sigma, \tau, \lambda, \delta, \alpha, \beta, m ; h)$.

Proof. For $f \in \mathcal{M}(\eta, \sigma, \tau, \lambda, \delta, \alpha, \beta, m ; h)$ and $g \in \mathcal{A}_{m}$, from (2.19) (used in the proof of Theorem 2.5), we can write

$$
\begin{aligned}
& \frac{1}{z}\left[\left(1-\frac{\eta(\tau+\lambda)}{(\lambda-\alpha) \beta+n \delta}\right) A_{\tau, \lambda, \delta}^{\sigma}(\alpha, \beta)(f * g)(z)+\frac{\eta(\tau+\lambda)}{(\lambda-\alpha) \beta+n \delta}\right. \\
& \left.\quad \times A_{\tau, \lambda, \delta}^{\sigma+1}(\alpha, \beta)(f * g)(z)\right] \\
& =\frac{g(z) *(z \varphi(z))}{g(z) * z} \quad(z \in U),
\end{aligned}
$$

where $\varphi(z)$ is defined as in (2.20). Since $h$ is convex univalent in $U, \psi(z) \prec h(z)$, $g(z) \in \operatorname{Re}(\alpha)$ and $z \in S^{*}(\alpha), \alpha<1$, it follows from (2.22) and Lemma 1.2, we obtain the result.

\section{REFERENCES}

[1] F. M. Al-Oboudi, On univalent functions defined by a generalized Sãlãgean operator, Int. J. Math. Math. Sci. 27 (2004), 1429-1436.

[2] A. Amourah and M. Darus, Some properties of a new class of univalent functions involving a new generalized differential operator with negative coefficients, Indian Journal of Science and Technology 9(36) (2016), 1-7.

[3] W. G. Atshan and A. K. Wanas, Differential subordinations of multivalent analytic functions associated with Ruscheweyh derivative, Analele Universitǎtii din Oradea - Fascicola Matematică XX(1) (2013), 27-33.

[4] N. E. Cho and T. H. Kim, Multiplier transformations and strongly close-to-convex functions, Bull. Korean Math. Soc. 40(3) (2003), 399-410.

[5] M. Darus and R. W. Ibrahim, On subclasses for generalized operators of complex order, Far East Journal of Mathematical Sciences 33(3) (2009), 299-308.

[6] P. L. Duren, Univalent Functions, Grundlehren der Mathematischen Wissenschaften, Band 259, Springer Verlag, New York, Berlin, Heidelberg, Tokyo, 1983.

[7] J. L. Liu, On a class of multivalent analytic functions associated with an integral operator, Bulletin of the Institute of Mathematics 5(1) (2010), 95-110.

[8] S. S. Miller and P. T. Mocanu, Differential subordinations and univalent functions, Michigan Math. J. 28 (1981), 157-171.

[9] J. K. Prajapat and R. K. Raina, Some applications of differential subordination for a general class of multivalently analytic functions involving a convolution structure, Math. J. Okayama Univ. 52 (2010), 147-158.

[10] S. Ruscheweyh, Convolutions in Geometric Function Theory, Les Presses de I'Université de Montréal, Montréal, 1982.

[11] G. St. Sălăgean, Subclasses of univalent functions, Lecture Notes in Mathematics 1013, Springer Verlag, Berlin, 1983, 362-372. 
[12] A. K. Wanas, Differential subordination results defined by new class for higher-order derivatives of multivalent analytic functions, International Journal of Advanced Research in Science, Engineering and Technology 4(8) (2017), 4363-4368.

[13] A. K. Wanas and A. H. Majeed, Differential subordinations for higher-order derivatives of multivalent analytic functions associated with Dziok-Srivastava operator, Analele Universitǎtii din Oradea - Fascicola Matematicǎ XXV(1) (2018), 33-42.

${ }^{1}$ Department of Mathematics,

College of Science, University of Al-QAdisiyah, IRAQ

Email address: abbas.kareem.w@qu.edu.iq 